\title{
Impedance of a Monopole Antenna With a Circular Con- ducting-Disk Ground System on the Surface of a Lossy Half Space
}

\author{
S. W. Maley and R. J. King
}

(September 27, 1960; revised December 1, 1960)

\begin{abstract}
The base impedance of a $\lambda / 4$ monopole antenna with a circular conducting disk ground system on the surface of a lossy half-space is calculated as a function of disk diameter using three different approximate methods. Verifying measurements were made on a model at $X$-band frequencies by simulating the lossy half-space with a water filled tank sufficiently large to approximate the results expected from an infinite lossy ground. The measured impedance values, $Z$, were broken up into the sum of two terms $\Delta Z$ and $Z^{\infty}$ where $Z^{\infty}$ is the impedance of the antenna with a perfectly conducting ground and $\Delta Z$ is a term which accounts for the finite size of the disk. The experimental measurements on a number of antennas indicate that the ratio $\Delta Z / Z^{\infty}$ as a function of disk diameter is nearly the same for all antennas measured. Comparison of the measured values of this ratio and the values calculated by the three different approximate methods indicate qualitative agreement.
\end{abstract}

\section{Introduction}

This paper presents a theoretical and experimental investigation of the impedance of a vertical monopole with a thin, circular, conducting disk ground system on the surface of a lossy half space as represented in figure 1 . The monopole impedance was calculated using three different methods and was experimentally measured for antenna heights near one-quarter wavelength and for a range of values of disk diameter. The investigation was made at $\mathrm{X}$-band frequencies to permit the experimental work to be done in the laboratory under carefully controlled conditions. The lossy half space was simulated by use of a water-filled tank about 120 wavelengths square. The calculated and measured values of impedance have been plotted on the same chart to permit comparison.

\section{Impedance Calculations}

\subsection{Formulation of Impedance}

The base impedance of the monopole antenna system represented in figure 1 is calculated using

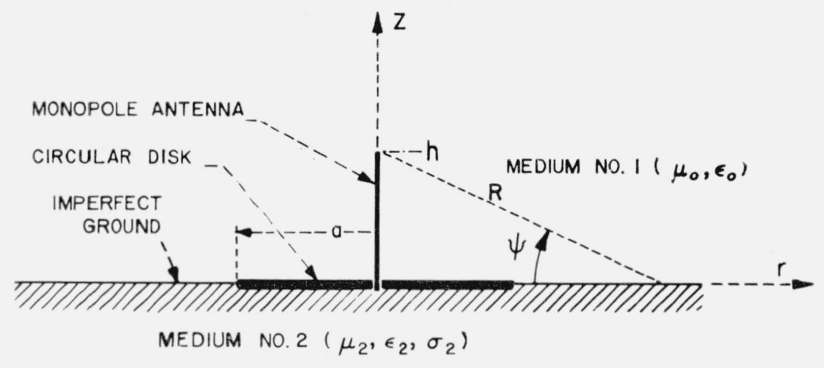

Figure 1. Monopole antenna with a circular conducting disk ground system on the surface of a lossy half-space.

${ }_{1}^{1}$ Contribution from Engineering Experiment Station, University of Colorado, Boulder, Colo. The work reported here was supported by the Electronics Research Directorate of the U. S. A ir Force Cambridge Research Center. three different approximate methods. Due to the symmetry of the configuration a cylindrical coordinate system is used making the field expressions independent of the azimuthal coordinate $\phi$.

It is convenient to express the magnetic field, $H_{\phi}$ $(r, z)$ in the form

$$
H_{\phi}(r, z)=H_{\phi}^{\infty}(r, z)+H_{\phi}^{s}(r, z) .
$$

The superscript " $\infty$ " denotes the field that would exist if the ground were perfectly conducting, while superscript " $s$ " denotes the difference between the actual field and the field that would exist for a perfectly conducting ground. We also express the antenna impedance $Z$ in a similar manner:

$$
Z=Z^{\infty}+\Delta Z \text {. }
$$

Here again the superscript " $\infty$ " denotes the impedance for a perfectly conducting ground, and $\Delta Z$ is the difference between the actual impedance and the impedance for a perfectly conducting ground.

As is shown by Wait and Pope, [5] ${ }^{2}$ the change in impedance, $\Delta Z$, may be expressed as

$$
\Delta Z=-\frac{1}{I_{0}^{2}} \int_{0}^{\infty} H_{\phi}^{\infty}(r, 0) E_{r}(r, 0) 2 \pi r d r
$$

$H_{\phi}^{\infty}(r, 0)$ is given by the expression

$$
H_{\phi}^{\infty}(r, 0)=-\frac{1}{2 \pi} \frac{\partial}{\partial r} \int_{0}^{h} \frac{e^{-j k\left(z^{2}+r^{2}\right)^{1 / 2}}}{\left(z^{2}-1 r^{2}\right)^{1 / 2}} I(z) d z,
$$

where

$$
k^{2}=\omega^{2} \mu_{0} \epsilon_{0} .
$$

In this expression, $I(z)$ is the current in the antenna as a function of $z$, and $I_{6}$ is the magnitude of the base current. The antenna is assumed to be thin and therefore the antenna current may be assumed real and sinusoidally distributed. Thus we have

2 Figures in brackets indicate the literature references at the end of this paper. 


$$
I(z)=\frac{I_{0}}{\sin \alpha} \sin (\alpha-k z) .
$$

$\alpha$ is given by

$$
\alpha=k\left(h+h^{\prime}\right),
$$

where $h$ is the antenna height, and $h^{\prime}$ is a parameter which characterizes the top loading. Using (5) eq (4) may be written as follows:

$$
H_{\phi}^{\infty}(r, 0)=-\frac{I_{0}}{2 \pi \sin \alpha} \frac{\partial}{\partial r} \int_{0}^{h} \frac{e^{-k j\left(z^{2}+r^{2}\right)^{1 / 2}}}{\left(z^{2}+r^{2}\right)^{1 / 2}} \sin (\alpha-k z) d z .
$$

The integration in eq (7) is straightforward but somewhat involved: the result is

$$
\begin{aligned}
H_{\phi}^{\infty}(r, 0) & =\frac{j I_{0}}{2 \pi \sin \alpha}\left[\frac{e^{-j k\left(r^{2}+h^{2}\right)^{1 / 2}}}{r} \cos (\alpha-k h)\right. \\
& \left.-\frac{e^{-j k r}}{r} \cos \alpha-\frac{j h e^{-j k\left(r^{2}+h^{2}\right)^{1 / 2}}}{\left(r^{2}+h^{2}\right)^{1 / 2} r} \sin (\alpha-k h)\right] .
\end{aligned}
$$

In order to evaluate eq (3), it is necessary to have an expression for $E_{r}(r, 0)$, but an exact solution for $E_{r}(r, 0)$ is not available in a useable form. Therefore, to obtain an answer, an approximation to $E_{r}$ $(r, 0)$ may be used to obtain an approximate value for $\Delta Z$. For low frequencies, Wait and Surtees [6] have used the approximate boundary condition given by

$$
\left.\begin{array}{rlrl}
E_{r}(r, 0) & \cong-\eta H_{\phi}(r, 0) & & r>a \\
& =0 & & r<a
\end{array}\right\},
$$

where

$$
\eta=\left(\frac{\mu_{2}}{\epsilon_{c}}\right)^{1 / 2}
$$

and

$$
\epsilon_{c}=\epsilon_{2}-j \frac{\sigma_{2}}{\omega}
$$

" $a$ " is the radius of the thin, circular conducting disk. Then they have used the additional approximation,

$$
H_{\phi}(r, 0) \cong H_{\phi}^{\infty}(r, 0) .
$$

Approximation (10) is valid for low frequencies but only for small values of $r$. However, at low frequencies, the significant contribution to the integral in (3) is for low values of $r$; therefore, the approximations (9) and (10) may both be used to give the approximation

$$
\left.\begin{array}{rlrl}
E_{r}(r, 0) & \cong-\eta H_{\phi}^{\infty}(r, 0) & & r>a \\
& =0 & & r<a
\end{array}\right\} .
$$

Another approximation suggested by Wait and Surtees is that given by

$$
\left.\begin{array}{rlrl}
E_{r}(r, 0) & \cong-\eta F(w) H_{\phi}^{\infty}(r, 0) & & r>a \\
& =0 & & r<a
\end{array}\right\},
$$

where $F(w)$ is the ground-wave attenuation factor as given by Norton [2]. For low frequencies and low values of $r$, (12) reduces to (11) because the factor $F(w)$ is very nearly unity with a zero phase angle. Approximation (12) is probably a better approximation than (11) for high frequencies.

For frequencies as high as that used in the accompanying experimental investigation $(10 \mathrm{kMc} / \mathrm{s})$, it was felt that a good approximation for $E_{r}(r, 0)$ would be that given by

$$
\left.\begin{array}{rlrl}
E_{r}(r, 0) \cong E_{r}^{\prime}(r, 0) & & r>a \\
& =0 & & r<a
\end{array}\right\},
$$

where $E_{r}^{\prime}(r, 0)$ is the radial component of the electric field at the surface of the ground for the case in which no disk is present. $E_{r}^{\prime}(r, 0)$ is given by Norton's formula (14).

\subsection{Calculation of Impedance}

Norton's formula [2] gives the tangential electric field at the surface of the ground for a current element of differential length $d l$, carrying a current $I$, and located a distance $b$, above the ground. Since this field is a function of $b$, the expression for the field will be written $E_{r}^{\prime}(r, 0, b)$ to indicate this dependence. Norton's formula may be written:

$$
\begin{aligned}
E_{r}^{\prime}(r, 0, b)= & -j 30 k I d l\left[\cos \psi\left(1-R_{v}\right) \frac{e^{-j k R}}{R}\right] \\
& \left\{\sin \psi+u\left(1-u^{2} \cos ^{2} \psi\right)^{1 / 2} F(w)\right. \\
& {\left[1-\frac{u^{2}\left(1-u^{2} \cos ^{2} \psi\right)}{2}+\frac{\sin ^{2} \psi}{2}+\frac{1}{j 2 k R}\right] } \\
+ & \left.\sin \psi \frac{1}{j k R}+u\left(1-u^{2} \cos ^{2} \psi\right)^{1 / 2} \frac{1}{j k R}\right\}
\end{aligned}
$$

where

$$
\begin{aligned}
R & =\left(r^{2}+b^{2}\right)^{1 / 2}, \\
u & =\frac{k}{k_{2}}
\end{aligned}
$$

and

$$
k_{2}^{2}=\omega^{2} \mu_{2} \epsilon_{c} .
$$

$R_{v}$ is the vertical reflection coefficient and $F(w)$ is the ground wave attenuation factor as given by Norton in the form of tables and charts [2]. $\epsilon_{c}$ is the complex permittivity of medium No. 2.

The field, $E_{r}(r, 0)$, is now given by

$$
E_{r}(r, 0) \cong E_{r}^{\prime}(r, 0)=\int_{0}^{h} E_{r}^{\prime}(r, 0, b) I(b) d b .
$$


The complexity of the expressions for $E_{r}^{\prime}(r, 0, b)$ makes it necessary to evaluate the integral in (15) numerically. This integral must be evaluated for each value of $r$ that is required. Since $E_{r}(r, 0)$ must be obtained by a numerical integration, eq (3) for $\Delta Z$ must also be evaluated by a numerical integration. The integral in (3) was approximated by a sum over a finite range of values of $r$; then a constant was added to correct for the use of the finite range for $r$ rather than an infinite range. It was found that this constant could be evaluated to a high degree of accuracy. Using this method it was found that the integrations in eqs (3) and (15) can be carried out numerically to an adequate degree of accuracy without a prohibitive amount of work. These calculations have been made for a quarter-wave monopole at $10 \mathrm{kMc} / \mathrm{s}$ for the case in which medium No. 2 is water. The complex dielectric constant used for water was $\epsilon_{c}=49-j 34$. The impedance $\Delta Z$ was also calculated for the same antenna and the same frequency, using the approximations (11) and (12).

The results of the calculations for all three approximations are shown along with the measured values, in figure 4 . The resistive and reactive components of $\Delta Z$ are plotted as functions of disk radius in wavelengths.

\section{Construction of the Experimental Equipment}

A large $12 \mathrm{ft} \times 12 \mathrm{ft} \times 4$ in. plywood water tank (117 wavelengths square at $9.60 \mathrm{kMc} / \mathrm{s}$ ) was held aloft so that the impedance measuring equipment could be placed directly beneath the monopole positioned at the tank center. It is anticipated that the finite size of the tank causes insignificant error because identical measurements were made with a tank roughly half the size of the one used here.

The monopole was fed by a $50 \Omega$ rigid air-coaxial line $0.620 \mathrm{in}$. in length which made a transition into 8 in. of $48 \Omega$ rigid air-coaxial line. The latter was in turn connected into a coaxial slotted section with a standard type " $\mathrm{N}$ " connector. A micrometer equipped probe carriage then provided a means of measuring the standing wave pattern position with a tolerance of $\pm 0.001 \mathrm{~cm}$.

The short section of $50 \Omega$ air-coaxial line used a replaceable center conductor which plugged into the $48 \Omega$ line center conductor. Various lengths of the monopole (an extension of the center conductor) could then be interchanged without disturbing the coaxial feed system. A single supporting teflon bead $0.020 \mathrm{in}$. long was fastened approximately midway in the $50 \Omega$ air-coaxial line. The inside diameter $(1 / 8$ in.) of the monopole feed line was made as small as machining considerations would allow, thereby better approximating a "thin" antenna and a "point" feed, and providing a very high attenuation $(75.3 \mathrm{db} / \mathrm{cm})$ for the $\mathrm{TE}_{11}$ mode of propagation.

Stability considerations required that the $48 \Omega$ line be as rigid as possible, thus allowing the monopole to be raised and lowered without disturbing the system calibration. For these same reasons, the number of mechanical joints was kept to a minimum.

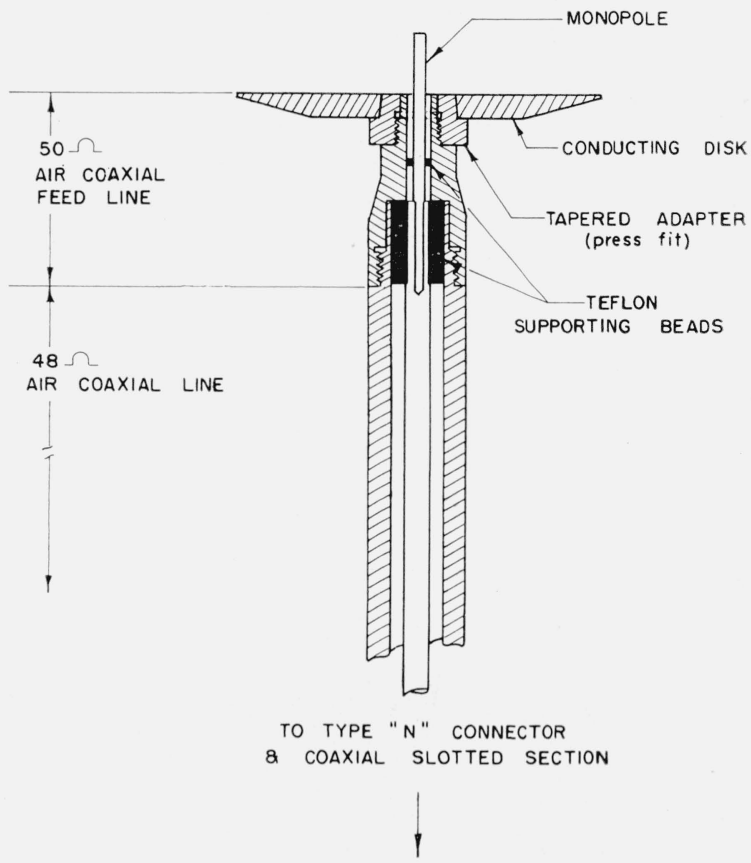

Figure 2. Assembled view of $0.750 \lambda$ radius disk with 0.250 $\lambda$ monopole and the associated coaxial feed line.

Although it was desirable to have a low residual standing wave ratio, the mechanical stability was far more important in achieving accurate calibration.

Measurements were taken for disks whose radii ranged from $1 / 8$ to $3 / 2$ wavelength and for monopoles with lengths from 0.230 to 0.270 wavelength and with a diameter of 0.045 wavelength. Data for the larger disks were taken using a single disk which was machined down about 0.050 in. in diameter after each successive measurement. This procedure provided accurate reseating of the disk after each radius change and presented a noticeable improvement over results obtained when a set of several disks of different radii was used. This machining procedure was continued to a disk radius of 1/4 wavelength; then three special disks were used to complete the range. The three smaller disks screw directly onto the $50 \Omega$ air-coax while the larger disk was set in place with the aid of an adapter. The adapter-disk connection utilized a press fit onto a slight taper. Figure 2 shows the cross section of the assembled feed line with the conducting disk mounted in position.

Ordinary tap water at $20^{\circ} \mathrm{C}$ was used as the surrounding imperfectly conducting ground region. The water meniscus at the edge of the conducting disk was eliminated by securing a $0.001 \mathrm{in}$. thickness of mylar sheet to the top surface with vacuumsystem grease. This established an abrupt transition between the disk and water. This grease is extremely viscous and has a high dielectric constant. The position of the disk could be accurately determined from the observer's position below the water tank with the aid of a special mirror-telescope arrangement. 


\section{Experimental Results}

The input reflection coefficient to the rigid coaxial line was measured as a function of the disk radius, and the results were plotted on a Smith Chart. The plot for a given monopole, as a function of disk radius, takes the shape of a spiral as shown in figure 3. Using an impedance transformation technique, which is described elsewhere, [4] the monopole base impedance was found; the resistive and reactive components were plotted as functions of disk radius. From these plots the impedance, $Z_{e}^{\infty}$, for an infinitely large disk could be estimated by extrapolation. This impedance then was subtracted from the monopole base impedance to give $\Delta Z$ as a function of disk radius.

Impedance measurements were made for a number of antennas. It was found that $Z_{e}^{\infty}$ varied appreciably with antenna length and further that it was somewhat higher in magnitude than would be expected on the basis of published analyses of a monepole over a perfectly conducting ground $[1,3]$. This discrepancy was thought to be due to the effects of the annular gap between the monopole and the circular conducting disk and of the high-frequency resistance of the monopole. It was found, however, that the ratio, $\Delta Z / Z_{e}^{\infty}$, plotted as a function of disk diameter was nearly the same for all antennas measured.

The experimentally established dependence of $\Delta Z$ upon $Z_{e}^{\infty}$ suggested that the function $\Delta Z / Z_{e}^{\infty}$ involving measured quantities $\Delta Z$ and $Z_{e}^{\infty}$ should be compared with the function $\Delta Z / Z_{c}^{\infty}$ involving the calculated function $\Delta Z$ and the calculated impedance, $Z_{c}^{\infty}$, of a monopole over a perfectly conducting ground. The value for $Z_{c}^{\infty}$ was taken to be $[1,3]$

$$
Z_{c}^{\infty}=34+j 16 .
$$

The function $\Delta Z / Z_{e}^{\infty}$, was measured and plotted for a number of antennas with lengths varying from 0.23 to 0.27 wavelengths. These plots were all nearly the same. Three representative plots for antenna lengths of $0.230,0.250$, and 0.270

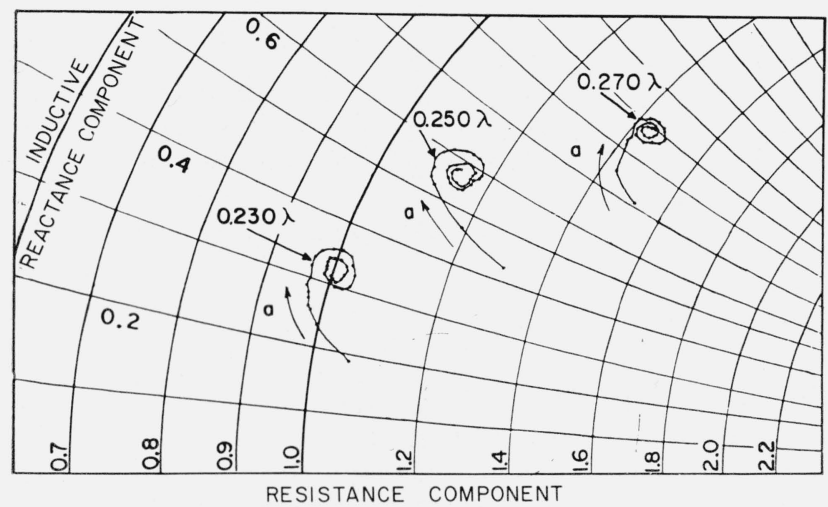

Figure 3. Measured monopole input reflection coefficient spirals for 3 monopole heights.

(Taken from a small section of a Smith chart). wavelength were selected and are shown in figure 4 . In order to present the results in terms of ohms rather than as dimensionless quantities, both the experimental functions $\Delta Z / Z_{e}^{\infty}$ and the calculated functions $\Delta Z / Z_{c}^{\infty}$ were multiplied by $Z_{c}^{\infty}$. The real parts of the resulting functions are labeled $\Delta R$ and the imaginary parts $\Delta X$.

The values of $Z_{e}^{\infty}$ for the three antennas for which measured data is presented are shown in table 1 .

TABLE 1 .

\begin{tabular}{|c|c|}
\hline $\begin{array}{l}\text { Monopole } \\
\text { height in } \\
\text { wavelengths }\end{array}$ & $7_{e}^{\infty}$ in ohms \\
\hline $\begin{array}{r}0.230 \\
.250 \\
.270\end{array}$ & $\begin{array}{l}45+j 6.3 \\
55.1+j 15.3 \\
77.7+j 24\end{array}$ \\
\hline
\end{tabular}

The calculations were made at $10 \mathrm{kMc} / \mathrm{s}$, but the experimental measurements were taken at 9.60 $\mathrm{kMc} / \mathrm{s}$. Since the radius of the disk is in wavelengths the curves may be compared directly.

\section{Conclusions}

Examination of the curves of figure 4 indicates that all three of the approximate methods of impedance calculation yield results which agree in a qualitative sense with the measured data in both amplitude and phase.

The theoretically calculated data was for a 0.25 $\lambda$ monopole; the measured data was for three different monopole heights, $0.23 \lambda, 0.25 \lambda$, and $0.27 \lambda$. The curves show that the measured data for the $0.23 \lambda$ monopole agree with the calculated data better than that for the $0.25 \lambda$ or $0.27 \lambda$ monopoles.

Comparing the individual calculated curves with measured curves shows that the approximation given by (11) is good for the resistive component of the impedance but is substantially too great in magnitude for the reactive component. The approximation given by (12) gives results which are too low in magnitude for both the resistive and reactive components; however, the phase in this case agrees well with the measured data. Of the three approximations considered, the one given by (14) seems to give results in closest agreement with the measured data in both amplitude and phase. These results show two things; first, that the approximation (14) is reasonably good; and second, that Norton's formula for the electric field tangential to the ground is reasonably accurate even for distance of the order of one wavelength from the antenna.

\section{References}

[1] R. W. P. King, The theory of linear antennas, p. 165 (Harvard University Press, Harvard, Conn., 1956)

[2] K. A. Norton, The propagation of radio waves over the surface of the earth and in the upper atmosphere, Proc. IRE 24, p. 1367 (Oct. 1936). 

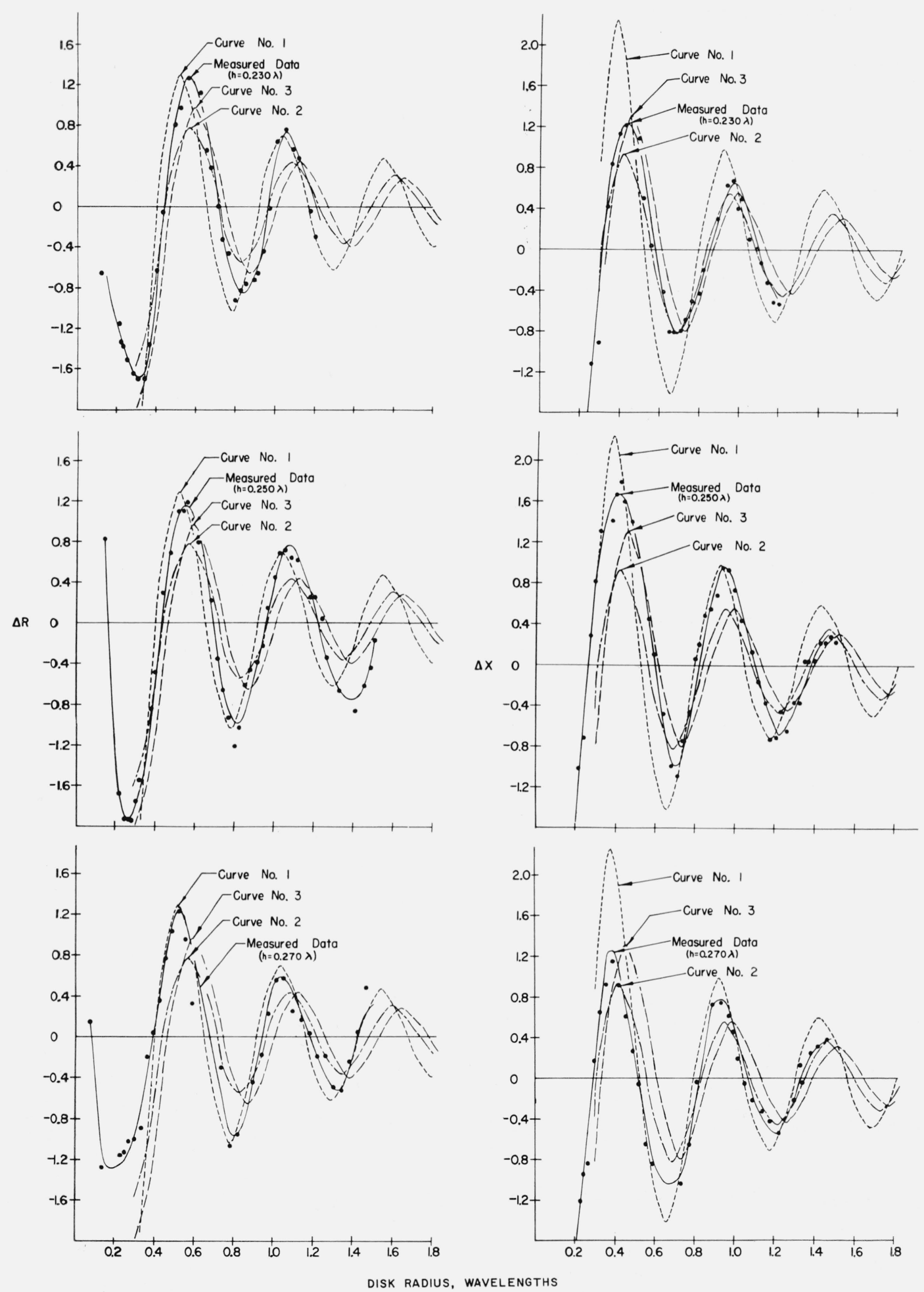

FIGURE 4. Calculated and measured values of incremental resistance and reactance of monopoles on a disk ground system as a function of disk radius.

The calculated curves are for a quarter-wave monopole at $10 \mathrm{kMc}$ and are based on the following approximations:

No. 3. $E_{r}(r, 0)$ as given by Norton's formula.

No. 2. $E_{\tau}(r, 0)=-\eta F_{(w)} H_{\phi}^{\infty}(r, 0)$

No. 1. $E_{r}(r, 0)=-\eta H_{\phi}^{\infty}(r, 0)$

The measured data is for a frequency of $9.60 \mathrm{kMc} / \mathrm{s}$ and a monopole height $h$. 
[3] S. Ramo and J. R. Whinnery, Fields and waves in modern radio, p. 546 (John Wiley \& Sons, Inc., New York, N.Y., 1953).

[4] Scientific Report ERD-TN-60-763, Impedance and pattern studies of disk-based monopoles over lossy ground surfaces, Electronics Research Directorate, Air Force Research Division, Air Research and Development Command, July 1960, Contract No. AF 19 (604) -4556.
[5] J. R. Wait and W. A. Pope, The characteristics of a vertical antenna with a radial conductor ground system, Applied Scientific Research, B 4, 177 (1954).

[6] J. R. Wait and W. J. Surtees, Impedance of a top loaded antenna of arbitrary length over a circular grounded screen, J. Appl. Phys. 25, 553 (1954).

(Paper 65D2-118) 